\title{
Influence of Chirality on Fluorescence and Resonance Energy Transfer
}

\author{
Kayn A. Forbes, David S. Bradshaw and David L. Andrews a) \\ School of Chemistry, University of East Anglia, Norwich NR4 7TJ, United Kingdom
}

\begin{abstract}
Electronically excited molecules frequently exhibit two distinctive decay mechanisms that rapidly generate optical emission: one is direct fluorescence; the other, energy transfer to a neighboring component. In the latter, the process leading to the ensuing 'indirect' fluorescence is known as FRET: fluorescence resonance energy transfer. For chiral molecules, both fluorescence and FRET exhibit discriminatory behavior with respect to optical and material handedness. While chiral effects such as circular dichroism are well known, as too is chiral discrimination for FRET in isolation, this article presents a study on a step-wise mechanism that involves both. Chirally sensitive processes follow excitation through the absorption of circularly polarized light, and are manifest in either direct or indirect fluorescence. Following recent studies setting down the symmetry principles, this analysis provides a rigorous, quantum outlook that complements and expands on these works. Circumventing expressions that contain complicated tensorial components, our results are amenable for determining representative numerical values for the relative importance of the various coupling processes. We discover that circular dichroism exerts a major influence on both fluorescence and FRET, and resolving the engagement of chirality in each component reveals the distinct roles of absorption and emission by, and between, donor and acceptor pairs. It emerges that chiral discrimination in the FRET stage is not, as might have been expected, the main arbiter in the step-wise mechanism. In the concluding discussion on various concepts, attention is focused on the validity of helicity transfer in FRET.
\end{abstract}

a)d.1.andrews@uea.ac.uk

\section{INTRODUCTION}

Chirality in optics has seen a surge in research effort in recent years, predominantly due to the novel effects connected to unique, fabricated nanostructures that are not found in nature.1-4 However, chiral interactions between light and molecules have been studied for centuries, with much of the underlying fundamental properties of chiroptical effects coming from this domain. ${ }^{5}$ In this sense it may seem that the prefix 'natural', often given to optical activity and circular dichroism, has never been more appropriate yet work on natural chiroptical effects involving light and conventional molecular matter is still proving a vital and fruitful testing ground. ${ }^{6}$ Recent examples include the separation of chiral molecules ${ }^{7-11}$ and the chiroptical effects of twisted light. ${ }^{12-14}$ It is known that light absorption by a chiral molecule, at different rates for left- and right-handed circularly polarized light, provides a foundation for the wellestablished spectroscopic technique called circular dichroism. ${ }^{15-17}$ The essentially time-reversed case that is based on fluorescence is, perhaps surprisingly, much less familiar, despite pioneering work on circularly polarized luminescence by Riehl and Richardson over 30 years ago. ${ }^{18,19}$

Incident light absorbed by a 'donor' molecule produces an excited state, the decay of which is often possible by two distinguishable mechanisms: direct fluorescence, or FRET fluorescence resonance energy transfer. The latter case delivers energy to an acceptor molecule, which may itself sub- sequently fluoresce. ${ }^{20,21}$ The issue of which is the most efficient route, for energy loss by the excited donor, depends on the distance to any prospective acceptor. If fluorescence occurs, a Stokes-shifted photon is emitted and the donor energy is dissipated in a random direction; if a suitable acceptor molecule is nearby, its excitation becomes possible via FRET. The excited acceptor may itself then emit a further Stokes-shifted photon, distinguishable from donor fluorescence due to its lower frequency. Studying the rate of donor fluorescence decrease and acceptor fluorescence increase is the basis for a 'spectroscopic ruler' - a quantitative measure of the distance between active sites. As such, the spectroscopic study of FRET ${ }^{22}$ has been proven as a tool to determine conformations of DNA, ${ }^{23}$ and FRET-based biosensors are pivotal in determining a plethora of biochemical activities, such as membrane potentials and intracellular ion levels. ${ }^{24-26}$ All of these uses are clinically important since they are performed optically, allowing the nanoscale study of live cells by non-invasive and non-destructive methods. ${ }^{27}$ FRET, of course, has many other applications including a relatively recent connection to synthetic self-assembled systems. ${ }^{28,} 29$

The use of FRET-based spectroscopy on biomolecules especially invites the appraisal of chirality in both fluorescence and FRET, since the vast majority of important biological macromolecules and pharmaceuticals are both fluorescent (usually under short-wavelength illumination) and chiral. There are indeed numerous reports of experimental 
studies specifically targeting the study and application of chiral discriminations in FRET and fluorescent emission see for example refs. 30-46. In terms of theory, however, beyond reports on the underlying principles of chirality in fluorescence, ${ }^{47-49}$ studies of chiral effects relevant to FRET are in short supply, being mostly limited to quantum electrodynamical (QED) studies of simple energy transfer between chiral species ${ }^{50-53}$ or within a chiral medium. ${ }^{54,55}$

In this article, the theory of fluorescence is developed to account for a sequence of events beginning with a chirally sensitive optical absorption process. For an electronic excited state that may then fluoresce, we examine the discriminatory chiroptical effects associated with direct fluorescence, and indirect fluorescence resulting from FRET to an acceptor molecule. Taking into account the full range of different optical sequences that may occur in the extended system, novel discriminatory effects are highlighted and compared. Obtaining detailed mechanisms for the differential rates of sequential optical and electrodynamical interactions, by our normal methods, leads to expressions of complicated tensorial form. At simplest, the implementation of these results for any specific system will lead to expressions with an intricate dependence on the relative orientations of the input beam, the intermolecular displacement vector, the direction of observation for the emitted fluorescence, and the internal orientational dispositions of the electric and magnetic transition moments for the donor and for the acceptor. These equations do not readily yield illustrative insights into the relative experimental significance of their multiple contributions. Thus, to secure representative numerical values that determine the relative importance of the various coupling mechanisms, we have resolved a scalar magnitude for each vector and tensor component. In the case of the electrodynamic coupling between the donor and the acceptor, we have been guided by well-known results for the associated coupling rates when averaged over all relative orientations. ${ }^{56}$ It emerges that chiral discrimination in the resonance energy transfer stage is not, as might have been considered, the main arbiter of chiral discrimination in FRET measurements: in this sense, the vaunted notion of 'chirality transfer' is essentially a nonstarter.

\section{DISCRIMINATORY FLUORESCENCE}

The light-molecule interactions involved in circularly polarized fluorescence (and the connected processes that follow) are properly described by a full quantum theory, in which electromagnetic radiation is quantized as well as the matter. ${ }^{57-60}$ In the following analysis, the decay of electronic excited states, subject to intramolecular vibrational losses, occurs through spin-allowed transitions, warranting the term 'fluorescence' (a pictorial representation of which is given by Figure 1) - though the formalism is also readily applied to the slower phosphorescence decay processes routed through spin-forbidden states. The cover-all term 'luminescence' appears almost as often in the literature and, in common with the large majority of optics, its theory is commonly cast in terms of the electric-dipole approximation. In most cases, this is adequate because transition electric dipole (E1) moments are much larger in magnitude than the next-largest contributions - those associated with magnetic dipole (M1) or electric quadrupole (E2) moments. However, where chirality is used, terms that involve electric dipoles alone often cancel out in chiral discrimination effects, as will be shown, and terms involving M1 and E2 need also to be considered. ${ }^{61,62}$ Nonetheless, in this work, E2 moments are ignored for two reasons: (a) if a fixed configuration for the system is chosen, the inclusion of E2 may as much as double the result, but will not introduce any new effects; (b) if the system is rotationally isotropic, then E2 contributions vanish. ${ }^{63}$

Developing theory from extensive symmetry groundwork reported elsewhere, ${ }^{64}$ we can begin with the matrix element (or quantum amplitude) $M_{\text {flu }}$ for circularly polarized fluorescence, accounting for the inclusion of both electric and magnetic dipole transitions. The key equation, derived using standard perturbation methods ${ }^{65}$ - is expressible in scalar form as follows;

$$
M_{\mathrm{flu}}^{(\mathrm{LLR})}=i\left(\frac{\hbar c k}{2 \varepsilon_{0} V}\right)^{\frac{1}{2}}\left\{\mu^{0 \alpha} \pm \frac{i m^{0 \alpha}}{c}\right\} \exp (-i k R)
$$

where $\mu^{0 \alpha}$ and $m^{0 \alpha}$ are the transition electric dipole and magnetic dipole moments (traversing from the excited state $\alpha$ to ground state 0 ), respectively, $k$ is the wave vector magnitude of the emission photon, $V$ is the quantization volume and $\exp (-\mathrm{i} k R)$ is the phase factor. Moreover, the second term of equation (1) involves a negative or a positive sign for the emission of left-handed (superscript L on $M_{\mathrm{flu}}$ ) or right-handed (R) circularly polarized light, respectively. The rate is then found from the Fermi Rule, $\Gamma=\left(2 \pi \rho_{\mathrm{f}} / \hbar\right)|M|^{2}$ where $\rho_{\mathrm{f}}$ is the density of final states. Hence, a first-order rate constant for circularly polarized fluorescence $k$ flu (the rate for a set of $N$ molecules) is determined from equation (1), giving;

$k_{\mathrm{flu}}^{(\mathrm{L} \mid \mathrm{R})}=\left(\frac{\pi c k \rho_{\mathrm{f}}}{\varepsilon_{0} V}\right)\left\{\left(\mu^{\alpha 0}\right)^{2} \mp \frac{2 i \mu^{\alpha 0} m^{\alpha 0}}{c}-\left(\frac{m^{\alpha 0}}{c}\right)^{2}\right\}$,

where, here and henceforth, $\mu^{0 \alpha}=\mu^{\alpha 0}=\bar{\mu}^{0 \alpha}=\bar{\mu}^{\alpha 0}$ and $m^{\alpha \alpha}=-m^{\alpha 0}=-\bar{m}^{\alpha \alpha}=\bar{m}^{\alpha 0}$ are employed - so that expressions that relate to either absorption or emission can be easily compared. The overbar denotes complex conjugation: the electric moments are real and the magnetic ones imaginary. ${ }^{63}$ Expression (2) is identical to the one used in circular dichroism studies, ${ }^{66}$ as is to be expected since emission is essentially time-reversed absorption. From equation (2) , and using $k^{(\mathrm{L})}-k^{(\mathrm{R})}$ to provide a result in terms of a difference in emission rate constants, we find the chiral discrimination as; 
$k_{\mathrm{flu}}^{\prime(\mathrm{L})}-k_{\mathrm{flu}}^{\prime(\mathrm{R})} \equiv k_{\mathrm{flu}}^{\prime}(\mathrm{L} \rightarrow \mathrm{R})=4 \operatorname{Im} \gamma$.

Here, the dimensionless parameter $\gamma=-m^{\alpha 0} / c \mu^{\alpha 0}$ and the prime on $k$ indicates normalization, meaning that they are divided by the leading term - i.e. the first term within the braces of equation (2) - to omit any pre-multipliers. Because the electric transition dipole moment $\mu^{\alpha 0}$ is space-odd and $m^{\alpha 0}$ space-even, inverting space leads to $\gamma$ changing sign: the effect of this inversion of space is equivalent to replacing one chiral molecule with its optical isomer, and therefore (3) is different for a right-handed molecule than for its left-handed enantiomeric equivalent. (In terms of fundamental symmetry, these two perspectives are akin to performing alibi and alias transformations.) Notice that the interference middle term of equation (2), involving both E1 and $\mathrm{M} 1$, is the only one that features in the differential rate. Also noteworthy is that, since electric and magnetic dipoles are real and imaginary quantities (respectively), the rate is correctly found to be a real amount. As a rule of thumb for optical transitions in molecular systems, $|\operatorname{Im}(\gamma)| \sim \alpha$, where $\alpha$ is the fine-structure constant $\sim 1 / 137$, and so we discover that the chiral discrimination given by equation (3) is roughly 20 times smaller than the leading contribution; namely, comparing the E1-M1 term with the E1-E1 term. Substantially higher levels of chiral selectivity become possible in plasmonic structures where magnetic dipole effects are more prominent. ${ }^{67}$

For purposes of the following, it is legitimately presumed that the electronic transition moments for the initial excitation $\alpha \leftarrow 0$ are similar in magnitude to those for an emission $0 \leftarrow \alpha^{\prime}$, meaning that expressions can be simplified because $\mu^{\alpha_{0}} \sim \mu^{0 \alpha^{\prime}}$ and $m^{\alpha_{0}} \sim-m^{0 \alpha^{\prime}}$. Here, the prime on the upper state for emission simply signifies that, within the same electronic state, the associated vibrational sub-level may be different from that which first results from optical excitation. Consistent with adoption of the Born-Oppenheimer approximation, this distinction allows for the fact that a degree of intramolecular vibrational decay will often be manifest in emission at a longer (Stokes-shifted) wavelength than the absorption. ${ }^{6}$

A rate constant for the two-step discriminatory circularly-excited fluorescence 'cef ' process (a pictorial representation of which is given by Figure 2) is determined from $k_{\text {cef }}=k_{\mathrm{abs}}^{(\mathrm{L} \mid \mathbb{R})} k_{\mathrm{flu}}^{(\mathrm{L} \mid \mathbb{R})}$, where $k_{\mathrm{abs}}$ is the rate constant for circularly excited absorption given by equation (2). Obviously four configurations are possible - LL, RR, LR and RL. However, we discover that the rate for left polarized input and righthanded emission (i.e. LR) is equivalent to that for right polarized input and left output (RL). For appropriate measures of chiral differentiation, we now find the differences in rate constants between distinct configurations, found using the same procedure as previously; these represent three different discrimination possibilities for 'cef ':

$k_{\text {cef }}^{\prime}(\mathrm{L} \rightarrow \mathrm{L})-k_{\text {cef }}^{\prime}(\mathrm{R} \rightarrow \mathrm{R})=8 \operatorname{Im} \gamma$, $k_{\text {cef }}^{\prime}(\mathrm{R} \rightarrow \mathrm{L})-k_{\mathrm{cef}}^{\prime}(\mathrm{R} \rightarrow \mathrm{R})=4 \operatorname{Im} \gamma+8 \gamma^{2}$,

$k_{\text {cef }}^{\prime}(\mathrm{L} \rightarrow \mathrm{L})-k_{\text {cef }}^{\prime}(\mathrm{R} \rightarrow \mathrm{L})=4 \operatorname{Im} \gamma-8 \gamma^{2}$,

where the notation $(\mathrm{L}|\mathrm{R} \rightarrow \mathrm{L}| \mathrm{R})$ denotes the handedness of the absorption step followed by $(\rightarrow)$ the handedness of the emitted photon. For example, equation (5) corresponds to the difference between a R absorption $\rightarrow$ L emission and a R absorption $\rightarrow \mathrm{R}$ emission at the chiral molecule clearly, in this case, the discrimination stems from the fluorescence process alone due to its change in handedness (following a similar argument, equation (6) arises from dichroism only).

While the terms linear in $\gamma$ represent the involvement of a magnetic dipole transition moment in either the absorption or the emission event, the above results also include chiroptical discrimination features up to the order of $\gamma^{2}$, signifying the potential involvement of a magnetic dipole in both events. For the many systems where terms beyond the linear approximation are negligible, the above results are consistent with the conclusions of a recent detailed symmetry analysis, ${ }^{64}$ based on that supposition. (Indeed the present analysis determines the specific value of $2 \operatorname{Im} \gamma$ for the 'discriminant' appearing in Table 2 of that paper). It should be noted that for molecules of intrinsically chiral form, $\gamma$ takes an opposite sign for each handedness of the material - i.e. each enantiomer.

The first differential rate, equation (4), is simply a sum of the circular dichroism and discriminatory fluorescence (which, as established above, are equal in value): in other words, this differential is double the circular dichroism result. In their linear terms, equations (5) and (6) are also consistent with (3) in accounting for chiral discrimination emission and absorption only once in each case, respectively; depending on the sign of the first term, the quadratic term either increases or diminishes the total discriminatory magnitude by a small amount. Being linearly dependent on $\gamma$, the first term in all the equations are dependent on the molecular handedness, as noted above, changing sign with a change of enantiomer - i.e. discriminatory florescence in (5) or circular dichroism in (6). However, the small additional effect in (5) and (6) with quadratic dependence $\gamma^{2}$ does not change sign for different enantiomers; the effect is solely due to the discriminatory interplay between the handedness of the input and output light.

\section{CIRCULARLY EXCITED FRET}

Extending the work of the previous section, we now analyze cases where the absorption and emission of circularly polarized light occur at physically distinct locations $A$ and $B$, with excitation shifting between them in an intervening step through resonance energy transfer (Figure 3). Overall we are dealing with a three-step process, which delivers a circularly excited FRET 'cet' rate given by the overall rate constant $k_{\mathrm{cet}}=k_{\mathrm{abs}}^{(\mathrm{L} / \mathrm{R})} k_{\mathrm{et}} k_{\mathrm{flu}}^{(\mathrm{LlR})}$. Here, $k_{\mathrm{et}}$ is the rate constant for FRET - the matrix element of which is given by; 
$M_{\mathrm{et}}=\mu_{\mathrm{A}}^{0 \alpha} \mu_{\mathrm{B}}^{\alpha 0} V+c^{-1}\left(\mu_{\mathrm{A}}^{0 \alpha} m_{\mathrm{B}}^{\alpha 0}+\mu_{\mathrm{B}}^{\alpha 0} m_{\mathrm{A}}^{0 \alpha}\right) U+c^{-2} m_{\mathrm{A}}^{0 \alpha} m_{\mathrm{B}}^{\alpha 0} V$,

where $V$ and $U$ denote electrodynamic coupling interactions between energy donor A and acceptor B. The detailed tensorial form of these interactions is provided elsewhere: 69 the former mediates E1-E1 and M1-M1 donor-acceptor interactions, the latter E1-M1 and M1-E1. FRET is generally dominated by the first term on the right-hand side of (7). However, the higher-order contributions, represented by the other terms, may become important in more complex systems, especially biological or nanoplasmonic structures, ${ }^{70,71}$ as deviations from the conventional FRET result are then seen to occur (when the ratio of molecular size to intermolecular separation is large, for example). ${ }^{72}$

Discriminatory effects are now identified in the sequence of absorption, followed by FRET and then fluorescence - i.e. indirect fluorescence. We determine that the following equation expresses the major contributions to the rate constant for FRET;

$k_{\text {et }}=\mu_{\mathrm{A}}^{2} \mu_{\mathrm{B}}^{2}|V|^{2}+2 c^{-2} \mu_{\mathrm{A}} m_{\mathrm{A}} \mu_{\mathrm{B}} m_{\mathrm{B}}\left(|U|^{2}-|V|^{2}\right)+\ldots \quad$,

where the first term relates to the dominant (E1-E1) 2 interaction, and the other two terms arising from interference correspond to discriminatory (E1-M1) ${ }^{2}$ and (M1-M1) (E1E1) couplings, respectively, both of which are dependent on the molecular handedness of A and B; all the superscripts $\alpha 0$ on $\mu$ and $m$ are now suppressed - their designation of transition moments is implicit. All other contributions are higher-order in terms of the number of magnetic interactions, and therefore insignificant. Of course, since there are two molecules in the system, the chiral differential equations now become substantially much more complicated. However, there are still only four rate combinations but, unlike the earlier systems, none are equal (due to the role of the molecular handedness of B), so that:

$$
k_{\text {cet }}^{\prime}\left(\mathrm{R}^{\mathrm{ET}} \mathrm{R}\right) \neq k_{\text {cet }}^{\prime}\left(\mathrm{R}^{\mathrm{ET}} \mathrm{L}\right) \neq k_{\text {cet }}^{\prime}(\mathrm{L} \stackrel{\mathrm{ET}}{\rightarrow} \mathrm{L}) \neq k_{\text {cet }}^{\prime}\left(\mathrm{L}^{\mathrm{ET}} \mathrm{R}\right),
$$

where 'ET' signifies the intervening energy transfer.

For measurements of chiral discrimination, it is once again primarily the differences in the rates for various kinds of polarization-resolved measurements that concerns us. We now have six distinct forms of chiral differential though, as shown in Table 1, there is only four separate results in the approximate form, of which any dissimilar pair constitutes a linearly independent basis. The full expressions of which are derived by multiplying equation (8) with the previous rates of cef; the exact chiral differentials are given in the Supplementary Material.

The full form of all six chiral differentials have a similar form; the leading contributions for one of them, for example, is given by:

$$
k_{\text {cet }}^{\prime}\left(\mathrm{L}^{\mathrm{ET}} \stackrel{\mathrm{L}}{)}-k_{\mathrm{cet}}^{\prime}(\mathrm{R} \stackrel{\mathrm{ET}}{\rightarrow} \mathrm{R})=4 \operatorname{Im}\left(\gamma_{\mathrm{A}}+\gamma_{\mathrm{B}}\right)\left[1+2 \gamma_{\mathrm{A}} \gamma_{\mathrm{B}}\left(\left|\frac{U}{V}\right|^{2}-1\right)\right] .\right.
$$

The magnitude of the quadratic ratio of couplings in the second term within the square brackets can be identified with the E1-E1 and E1-M1 transfer rates, represented as the respective scalars $A(k, R)=V \bar{V}$ and $B(k, R)=U \bar{U}$ derived in ref. 69. As such, we have;

$$
\left|\frac{U}{V}\right|^{2} \sim \frac{k^{2} R^{2}+k^{4} R^{4}}{3+k^{2} R^{2}+k^{4} R^{4}} \approx \frac{1}{3} k^{2} R^{2}=\frac{4}{3} \pi^{2} \lambda^{2} R^{2},
$$

where the right-hand side of this expression focuses on couplings over short-range separations of A and B, i.e. distances smaller than the reduced wavelength for the transfer energy, such that $k R \ll 1$. This condition is almost invariably satisfied in systems where the effects of energy transfer are measurable. Based on data values for wavelengths $\lambda$ and donor-acceptor distances $R$ provided in a well-known review, ${ }^{73}$ typical values for the right-hand side of equation (11) would be negligibly small, in the region of $10^{-4}-10^{-7}$. Thus the square brackets effectively reduce to $1-2 \gamma_{A} \gamma_{B}$, in which the product of $\gamma$ parameters also has values in the same small range. Thus, the result from equation (10) effectively reduces to $4 \operatorname{Im}\left(\gamma_{A}+\gamma_{B}\right)$, as indicated by the corresponding entry in Table 1 . It is noteworthy that the same result arises in the long-range, since equation (11) equates to unity. Each of its entries represent a form of differential effect that may, in principle, be measured as a function of input or output wavelength, or indeed as a function of both in a novel form of 2D chiroptical spectroscopy. Similar considerations apply to the other, simplified results given in this Table.

There are several notable features of Table 1, with its appealing simplicity. For example, in the case where A = B, i.e. the donor and the acceptor are chemically equivalent and both either right- or left-handed molecules, the equivalent result to equation (4) is obtained:

$k_{\text {cet }}^{\prime}\left(\mathrm{L}^{\mathrm{ET}} \mathrm{L}\right)-k_{\text {cet }}^{\prime}\left(\mathrm{R}^{\mathrm{ET}} \mathrm{R}\right)=8 \operatorname{Im} \gamma$,

the result clearly being dominated by the sum of discriminatory absorption and fluorescence. Conversely, if A and B were to be opposite enantiomers the result would be zero correctly signifying that the composite pair is achiral. Again, if $\mathrm{A}=\mathrm{B}$, then $k_{\text {cet }}^{\prime}(\mathrm{LTT} \rightarrow \mathrm{R})=k_{\text {cet }}^{\prime}\left(\mathrm{RT}^{\mathrm{ET}} \mathrm{L}\right)$ and the difference between the two measurements vanishes. Now consider the pair $k_{\text {cet }}^{\prime}\left(\mathrm{L}^{\mathrm{ET}} \stackrel{\mathrm{L}}{\rightarrow}\right)$ and $k_{\text {cet }}^{\prime}\left(\mathrm{R}^{\mathrm{ET}} \rightarrow \mathrm{L}\right)$, whose difference in rate constant depends (to this level of approximation) only on A: if A is achiral then the difference disappears. This contrasts with induced circular dichroism, ${ }^{63}$ based on the input 
of circularly polarized light whose wavelength is off-resonant with $A$, through which in a concerted mechanism there can result a difference in the rate of excitation of $B$ - which will, in turn, be evident in the absolute rate of fluorescence from $B$.

In the development of broader studies of chiral interaction, the case of discriminatory FRET in isolation is worth comparison. ${ }^{50,53}$ Analyzing equation (8), for example, the contribution that dominates FRET is the first term, which relates to E1-E1 coupling between the donor and acceptor - in most FRET studies, any other terms need not be included. The second and third terms correspond to E1-M1 interactions (dependent on $|U|^{2}$ ) and the interference between E1-E1 and M1-M1 couplings (relating to $|V|^{2}$ ), respectively. These latter two contributors apply to chiralitysensitive FRET: an interference between pure electric and pure magnetic terms, and a mixed electric-magnetic contribution. Usually, when considering chirality effects in FRET, these two terms are significant. Nevertheless, we have shown that energy transfer between the donor and acceptor plays a minor role in the chiral differentials of circularly excited FRET. In particular, the electrodynamic couplings $U$ between the magnetic transition dipole of the donor with an electric transition moment of the acceptor, or vice versa (one of the leading orders in chirality-sensitive FRET) prove not to be engaged to a significant extent.

\section{DISCUSSION}

While, in previous work, chirality connected to absorption, fluorescence and energy transfer have been studied in isolation, this is the first attempt at rigorously examining a combination of all three in a single process that is both stepwise and linear. Since the processes we have considered depend linearly on optical input, there is no simple connection to recent studies on chirality-sensitive nonlinear spectroscopy. ${ }^{74-78}$ In applying the results that our analysis has secured, it is first to be noted that, for any specific enantiomer, circular dichroism exhibits dispersive behavior, and any associated measure of chirality necessarily varies with the wavelength of light and the specific electronic state excited by optical absorption. Therefore, we should emphasize that the $\gamma$ parameters are specifically defined for each excited state of the molecule to which they relate. As such, for each wavelength, Im $\gamma_{A}$ and $\operatorname{Im} \gamma_{B}$ serve as crude but effective measures of the chirality of each component - however, as now described, there is no universal measure.

It is widely known that optical helicity can only be conveyed in electrodynamical form through association with angular momentum. ${ }^{79}$ Nonetheless, notwithstanding the close connection between angular momentum and chirality (most obvious in the relationship between spin angular momentum and circular polarizations), ${ }^{80}$ there is no universal measure of chirality applicable to both light and matter. ${ }^{6}$ Indeed, as has been shown by detailed multipolar analysis, ${ }^{81}$ angular momentum is not, in general, a conserved quantity in resonance energy transfer between molecules with closed shell electron configurations. The quantization of that angular momentum is only valid along a single axis, which in the case of light identifies with the propagation vector. Therefore, the seemingly attractive prospect of helicity transfer in FRET ${ }^{82,83}$ is not supported by fundamental principles.

We now provide brief comment on some recent innovative experimental investigations on chirality. ${ }^{84,85}$ It is evident, in these reports, that the concepts of 'chirality transfer' or 'chiral energy' are deployed as descriptors. However, such terms are misleading and we suggest their usage is inadvisable. Each phrase suggests a proven mechanism that is, in fact, physically baseless. First, the term 'chirality' is meaningful only when used with reference to a specific region and dimension of space. There is no scaling or conservation law for chirality when the term is applied to physical matter. Secondly, energy is specifically defined as a scalar physical quantity, with no connection to any vectorial frame. It is understandable that when energy transfer results in manifestations of chirality by an acceptor, cognate with effects in the donor, that it might seem chirality in some sense accompanies the energy transfer process. However, the fundamental electrodynamics does not support such an interpretation. At the root of the energy transfer process is an unweighted sum over virtual photons of any two independent polarizations. Left- and right-handed virtual photons contribute equally to the calculation that leads directly to the familiar coupling tensors, responsible for the transfer process. This proves that no sense of circularity can be imparted from any donor to an acceptor, in the course of energy transfer.

In this article, we have determined from all the possible optical sequences studied in the course of fluorescence, FRET, and the circularly-excited forms of both, that there can be a plethora of distinct individual contributions involved. However, the dominant contribution to any of these effects is solely due to either the discriminatory single-photon absorption or emission event, or the combined effect of the two. This means that, compared to discriminatory fluorescence in isolation, the chiral differential signal can be as much as doubled when circularly polarized light absorption is included. None of the many novel (and smaller in magnitude) chiroptical mechanisms in any of the FRET processes meaningfully increases (or decreases) the overall discriminatory signal - though they do deliver different physics. Significantly, this proves that chirality can in no way be considered to transfer across the system, as all the effects reduce to the dominant and well-known optical processes of photon absorption and emission. It may, thus, be asserted that there exists no special route to increase any chiroptical effect of fluorescence or FRET in molecular systems by considering the processes as a concerted sequence: the single photon physics at play always dominates, in consistency with every aspect of fundamental electrodynamics. 


\section{SUPPLEMENTARY MATERIAL}

See supplementary material for the complete form of the chiral differentials, which include higher-order contributions, and the derivation of equation (8).

\section{REFERENCES}

1 Z. Li, M. Mutlu, and E. Ozbay, J. Opt. 15, 023001 (2013).

2 Z. Wang, F. Cheng, T. Winsor, and Y. Liu, Nanotechnology 27, 412001 (2016).

3 P. Lodahl, S. Mahmoodian, S. Stobbe, A. Rauschenbeutel, P. Schneeweiss, J. Volz, H. Pichler, and P. Zoller, Nature 541, 473 (2017).

4 J. T. Collins, C. Kuppe, D. C. Hooper, C. Sibilia, M. Centini, and V. K. Valev, Adv. Opt. Mater. 5, 1700182 (2017).

5 S. F. Mason, Molecular Optical Activity and the Chiral Discriminations (Cambridge University Press, Cambridge, 1982).

6 D. S. Bradshaw, J. M. Leeder, M. M. Coles, and D. L. Andrews, Chem. Phys. Lett. 626, 106 (2015).

7 A. Canaguier-Durand, J. A. Hutchison, C. Genet, and T. W. Ebbesen, New J. Phys. 15, 123037 (2013).

8 G. Tkachenko and E. Brasselet, Nat. Commun. 5, 3577 (2014).

9 R. P. Cameron, S. M. Barnett, and A. M. Yao, New J. Phys. 16, 013020 (2014).

${ }^{10}$ D. S. Bradshaw and D. L. Andrews, New J. Phys. 16, 103021 (2014).

${ }^{11}$ Y. Zhao, A. A. E. Saleh, and J. A. Dionne, ACS Photonics 3, 304 (2016).

${ }^{12}$ A. Afanasev, C. E. Carlson, and M. Solyanik, J. Opt. 19, 105401 (2017).

${ }^{13}$ K. A. Forbes and D. L. Andrews, Phys. Rev. A 99, 023837 (2019).

${ }^{14}$ K. A. Forbes, Phys. Rev. Lett. 122, 103201 (2019).

${ }^{15}$ G. Gottarelli, S. Lena, S. Masiero, S. Pieraccini, and G. P. Spada, Chirality 20, 471 (2008).

${ }^{16}$ G. Siligardi, R. Hussain, S. G. Patching, and M. K. Phillips-Jones, Biochim. Biophys. Acta 1838, 34 (2014).

${ }^{17}$ A. J. Miles and B. A. Wallace, Chem. Soc. Rev. 45, 4859 (2016).

${ }^{18}$ J. P. Riehl and F. S. Richardson, Chem. Rev. 86, 1 (1986).

${ }^{19}$ J. P. Riehl and F. S. Richardson, Methods Enzymol. 226, 539 (1993).

${ }^{20}$ D. L. Andrews and A. A. Demidov, Resonance Energy Transfer (Wiley, Chichester, 1999).

${ }^{21}$ D. M. Jameson, Introduction to Fluorescence (CRC Press, Boca Raton, 2014).

${ }^{22}$ D. W. Piston and G.-J. Kremers, Trends Biochem. Sci 32, 407 (2007).

${ }^{23}$ R. Vafabakhsh and T. Ha, Science 337, 1097 (2012).

${ }^{24}$ A. Miyawaki, Annu. Rev. Biochem 80, 357 (2011).

${ }^{25}$ A. J. Lam, F. St-Pierre, Y. Gong, J. D. Marshall, P. J. Cranfill, M. A. Baird, M. R. McKeown, J. Wiedenmann, M. W. Davidson, M. J. Schnitzer, R. Y. Tsien, and M. Z. Lin, Nature Methods 9, 1005 (2012).

${ }^{26}$ P. Nelson, From Photon to Neuron: Light, Imaging, Vision (Princeton University Press, Princeton, 2017).

${ }^{27}$ B. T. Bajar, E. S. Wang, S. Zhang, M. Z. Lin, and J. Chu, Sensors 16, 1488 (2016).
${ }^{28}$ E. Maligaspe, T. Kumpulainen, H. Lemmetyinen, N. V. Tkachenko, N. K. Subbaiyan, M. E. Zandler, and F. D'Souza, J. Phys. Chem. A 114, 268 (2010)

${ }^{29}$ V. K. Praveen, C. Ranjith, E. Bandini, A. Ajayaghosh, and N. Armaroli, Chem. Soc. Rev. 43, 4222 (2014).

${ }^{30}$ D. H. Metcalf, S. W. Snyder, J. Demas, and F. Richardson, J. Am. Chem. Soc. 112, 5681 (1990).

${ }^{31}$ D. H. Metcalf, J. P. Bolender, M. S. Driver, and F. Richardson, J. Phys. Chem. 97, 553 (1993).

32 J. E. Field, G. Muller, J. P. Riehl, and D. Venkataraman, J. Am. Chem. Soc. 125, 11808 (2003).

${ }^{33}$ J. Zhao and T. D. James, Chem. Commun., 1889 (2005).

${ }^{34}$ J. P. Riehl and G. Muller, in Comprehensive Chiroptical Spectroscopy: Instrumentation, Methodologies, And Theoretical Simulations2011), pp. 65.

${ }^{35}$ J. Liu, H. Su, L. Meng, Y. Zhao, C. Deng, J. C. Ng, P. Lu, M. Faisal, J. W. Lam, and X. Huang, Chem. Sci. 3, 2737 (2012).

${ }^{36}$ E. M. Sánchez-Carnerero, A. R. Agarrabeitia, F. Moreno, B. L. Maroto, G. Muller, M. J. Ortiz, and S. de la Moya, Chem. Eur. J. 21, 13488 (2015).

${ }^{37}$ B. Le Feber, N. Rotenberg, and L. Kuipers, Nat. Commun. 6, 6695 (2015).

${ }^{38}$ S. Lobanov, S. Tikhodeev, N. Gippius, A. Maksimov, E. Filatov, I. Tartakovskii, V. Kulakovskii, T. Weiss, C. Schneider, and J. Geßler, Phys. Rev. B 92, 205309 (2015).

${ }^{39}$ M. Cotrufo, C. I. Osorio, and A. F. Koenderink, ACS Nano 10, 3389 (2016).

${ }^{40}$ G. Longhi, E. Castiglioni, J. Koshoubu, G. Mazzeo, and S. Abbate, Chirality 28, 696 (2016).

${ }^{41}$ M. Nakamura, J. Suzuki, F. Ota, T. Takada, K. Akagi, and K. Yamana, Chem. Eur. J. 22, 9121 (2016).

${ }^{42}$ L. Cerdan, F. Moreno, M. Johnson, G. Muller, S. de la Moya, and I. Garcia-Moreno, Phys. Chem. Chem. Phys. 19, 22088 (2017).

${ }^{43}$ J. Jiménez, L. Cerdán, F. Moreno, B. L. Maroto, I. García-Moreno, J. L. Lunkley, G. Muller, and S. de la Moya, J. Phys. Chem. C 121, 5287 (2017)

${ }^{44}$ S. Takahashi, Y. Ota, T. Tajiri, J. Tatebayashi, S. Iwamoto, and Y. Arakawa, Phys. Rev. B 96, 195404 (2017).

${ }^{45}$ H.-T. Lin, C.-Y. Chang, P.-J. Cheng, M.-Y. Li, C.-C. Cheng, S.-W. Chang, L. L. J. Li, C.-W. Chu, P.-K. Wei, and M.-H. Shih, ACS Appl. Mater. Interfaces 10, 15996 (2018).

${ }^{46}$ H. Zheng, W. Li, W. Li, X. Wang, Z. Tang, S. X. A. Zhang, and Y. Xu, Adv. Mater. 30, 1705948 (2018).

${ }^{47}$ J. M. Leeder, H. T. Haniewicz, and D. L. Andrews, J. Opt. Soc. Am. B 32, 2308 (2015).

48 J. M. Leeder, H. T. Haniewicz, and D. L. Andrews, Proc. SPIE 9545 , 94502 (2015).

${ }^{49}$ F. Crimin, N. Mackinnon, J. B. Götte, and S. M. Barnett, Applied Sciences 9, 828 (2019).

${ }^{50}$ D. Craig and T. Thirunamachandran, J. Chem. Phys. 109, 1259 (1998).

${ }^{51}$ D. Craig and T. Thirunamachandran, Theor. Chem. Acc. 102, 112 (1999).

${ }^{52}$ A. Salam, J. Chem. Phys. 122, 044112 (2005).

${ }^{53}$ A. Salam, Atoms 6, 56 (2018).

${ }^{54}$ J. J. Rodriguez and A. Salam, Chem. Phys. Lett. 498, 67 (2010).

55 J. J. Rodriguez and A. Salam, J. Phys. Chem. B 115, 5183 (2010). 
${ }^{56}$ G. J. Daniels, R. D. Jenkins, D. S. Bradshaw, and D. L. Andrews, J. Chem. Phys. 119, 2264 (2003).

${ }^{57}$ D. P. Craig and T. Thirunamachandran, Molecular Quantum Electrodynamics: An Introduction to Radiation-Molecule Interactions (Dover Publications, Mineola, NY, 1998).

${ }^{58}$ I. Lindgren, S. Salomonson, and D. Hedendahl, Int. J. Quant. Chem. 108, 2272 (2008).

${ }^{59}$ A. Salam, Molecular Quantum Electrodynamics: Long-Range Intermolecular Interactions (Wiley, Hoboken, NJ, 2009).

${ }^{60}$ D. L. Andrews, G. A. Jones, A. Salam, and R. G. Woolley, J. Chem. Phys. 148, 040901 (2018).

${ }^{61}$ L. D. Barron, Molecular Light Scattering and Optical Activity (Cambridge University Press, Cambridge, 2009).

${ }^{62}$ D. L. Andrews, J. Opt. 20, 033003 (2018).

${ }^{63}$ D. P. Craig and T. Thirunamachandran, Molecular Quantum Electrodynamics: An Introduction to Radiation-Molecule Interactions (Dover Publications, Mineola, NY, 1998).

${ }^{64}$ D. L. Andrews, Methods Appl. Fluoresc. 7, 032001 (2019).

${ }^{65}$ D. S. Bradshaw and D. L. Andrews, J. Phys. Chem. A 117, 75 (2013).

${ }^{66}$ E. A. Power and T. Thirunamachandran, J. Chem. Phys. 60, 3695 (1974).

${ }^{67}$ V. K. Valev, J. J. Baumberg, C. Sibilia, and T. Verbiest, Adv. Mater. 25, 2517 (2013).

${ }^{68}$ D. L. Andrews, Molecular Photophysics and Spectroscopy (Morgan \& Claypool Publishers, San Rafael, CA, 2014).

${ }^{69}$ D. L. Andrews and D. S. Bradshaw, Eur. J. Phys. 25, 845 (2004).

${ }^{70}$ M. R. Bourgeois, A. T. Liu, M. B. Ross, J. M. Berlin, and G. C. Schatz, J. Phys. Chem. C 121, 15915 (2017).

${ }^{71}$ K. Nasiri Avanaki, W. Ding, and G. C. Schatz, J. Phys. Chem. C 122, 29445 (2018).
${ }^{72}$ G. D. Scholes, R. D. Harcourt, and G. R. Fleming, J. Phys. Chem. B 101, 7302 (1997).

${ }^{73}$ C. G. dos Remedios and P. D. J. Moens, in Resonance Energy Transfer, edited by D. L. Andrews, and A. A. Demidov (Wiley, New York, 1999), pp. 1.

${ }^{74}$ G. J. Simpson, ChemPhysChem 5, 1301 (2004).

${ }^{75} \mathrm{P}$. Fischer and F. Hache, Chirality 17, 421 (2005).

${ }^{76}$ M. J. Huttunen, G. Bautista, M. Decker, S. Linden, M. Wegener, and M. Kauranen, Opt. Mater. Express 1, 46 (2011).

${ }^{77}$ E. A. Mamonov, T. V. Murzina, I. A. Kolmychek, A. I. Maydykovsky, V. K. Valev, A. V. Silhanek, T. Verbiest, V. V. Moshchalkov, and O. A. Aktsipetrov, Opt. Express 20, 8518 (2012).

${ }^{78}$ S. P. Rodrigues, S. Lan, L. Kang, Y. Cui, and W. Cai, Adv. Mater. 26, 6157 (2014).

${ }^{79}$ K. Y. Bliokh, A. Y. Bekshaev, and F. Nori, New J. Phys. 15, 033026 (2013).

${ }^{80}$ I. Fernandez-Corbaton and C. Rockstuhl, Phys. Rev. A 95, 053829 (2017).

${ }^{81}$ D. L. Andrews, Phys. Rev. A 81, 033825 (2010).

${ }^{82}$ M. Nieto-Vesperinas, Phys. Rev. A 92, 023813 (2015).

${ }^{83}$ L. Bene, M. Bagdány, and L. Damjanovich, Biophys. Chem. 239, 38 (2018).

${ }^{84}$ D. Yang, P. Duan, L. Zhang, and M. Liu, Nat. Commun. 8, 15727 (2017).

${ }^{85}$ L. Ji, Y. Sang, G. Ouyang, D. Yang, P. Duan, Y. Jiang, and M. Liu, Angew. Chem. Int. Ed. 58, 844 (2019). 


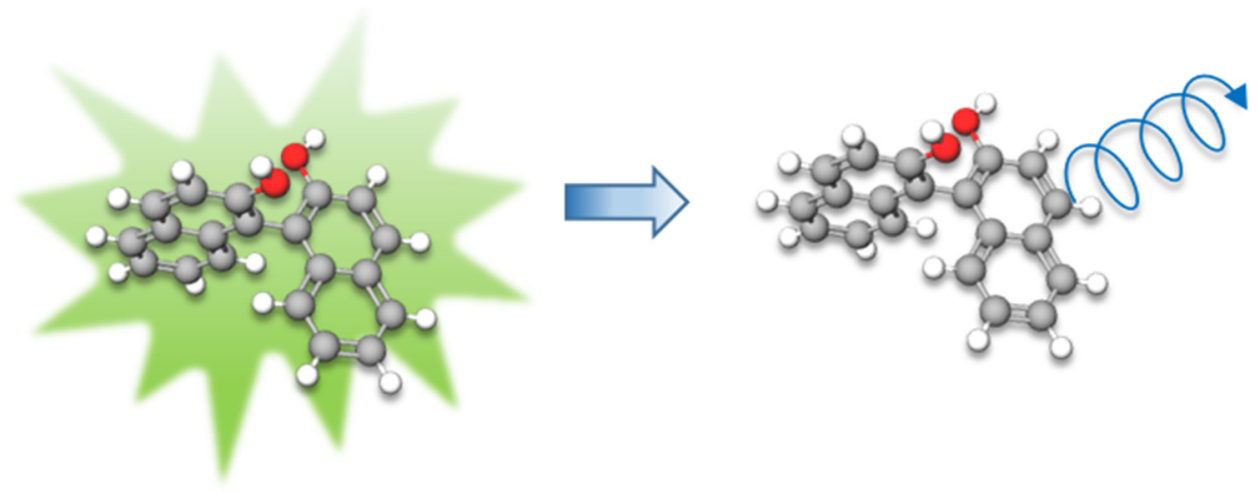

Figure 1. Emission of circularly polarized light (blue spiral arrow), in the form of fluorescence, from an excited chiral molecule.

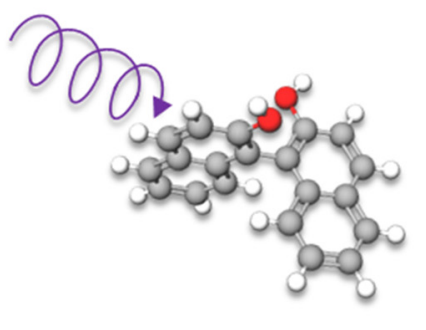

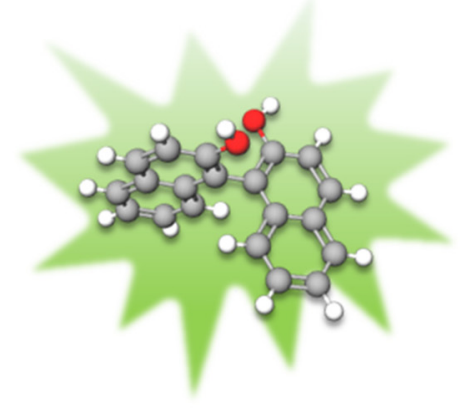

(a)

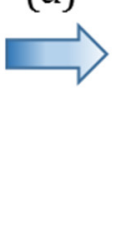

(b)

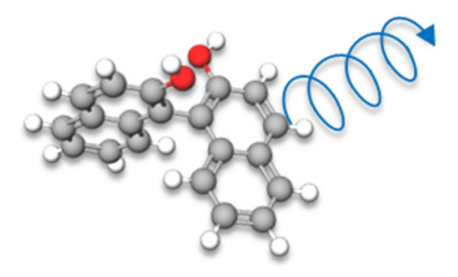

Figure 2. Representation of step-wise circularly excited fluorescence: (a) a chiral molecule is excited via absorption of circularly polarized light (purple spiral arrow), (b) excited chiral molecule emits circularly polarized light (blue arrow). Absorption and emission frequencies may be different due to internal relaxation between steps (not shown). 


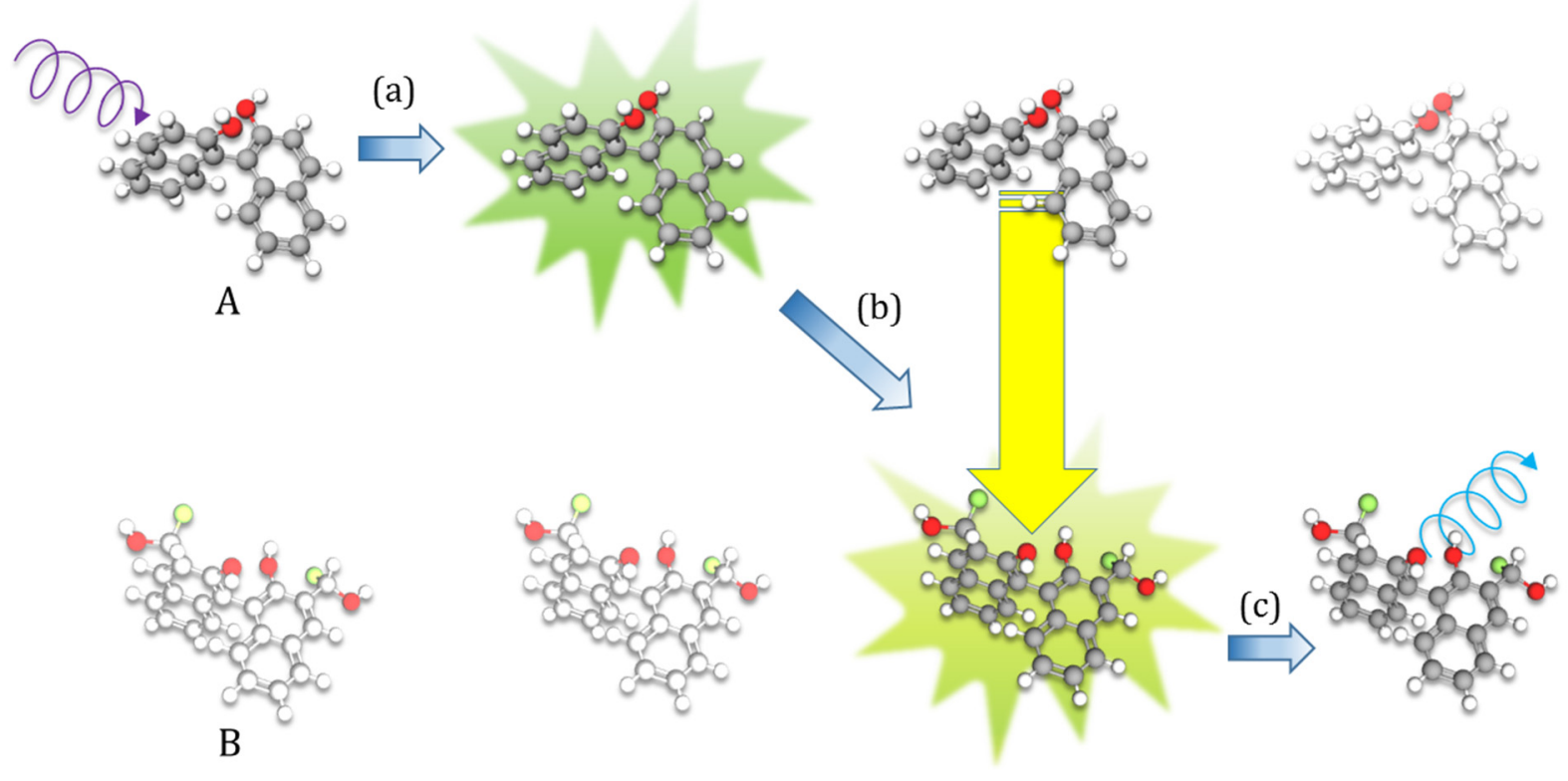

Figure 3. Representation of step-wise circularly excited FRET: (a) a chiral donor, A, is excited via absorption of circularly polarized light (purple spiral arrow), (b) energy is transferred from A to chiral acceptor B, (c) excited B emits circularly polarized light (blue arrow). Light absorption at $\mathrm{A}$ and emission at $\mathrm{B}$ may have different frequencies due to relaxation during the excited state lifetimes of A and B (not shown).

\begin{tabular}{|c|c|c|c|}
\hline $\begin{array}{c}\text { Measurable } 1 \\
\downarrow\end{array}$ & & & \\
\hline$k_{\text {cet }}^{\prime}\left(\mathrm{R}^{\mathrm{ET}} \rightarrow \mathrm{L}\right)$ & $4 \operatorname{Im} \gamma_{\mathrm{B}}$ & & \\
\hline$k_{\text {cet }}^{\prime}\left(\mathrm{L}^{\mathrm{ET}} \rightarrow \mathrm{L}\right)$ & $4 \operatorname{Im}\left(\gamma_{A}+\gamma_{B}\right)$ & $4 \operatorname{Im} \gamma_{\mathrm{A}}$ & \\
\hline$k_{\text {cet }}^{\prime}\left(\mathrm{L}^{\mathrm{ET}} \rightarrow \mathrm{R}\right)$ & $4 \operatorname{Im} \gamma_{\mathrm{A}}$ & $4 \operatorname{Im}\left(\gamma_{\mathrm{A}}-\gamma_{\mathrm{B}}\right)$ & $4 \operatorname{Im} \gamma_{\mathrm{B}}$ \\
\hline$\underset{\rightarrow}{\text { Measurable } 2}$ & $k_{\mathrm{et}}^{\prime}\left(\mathrm{R}^{\mathrm{ET}} \rightarrow \mathrm{R}\right)$ & $k_{\text {cet }}^{\prime}\left(\mathrm{R}^{\mathrm{ET}} \rightarrow \mathrm{L}\right)$ & $k_{\text {cet }}^{\prime}\left(\mathrm{L}^{\mathrm{ET}} \rightarrow \mathrm{L}\right)$ \\
\hline
\end{tabular}

Table I. Approximate differences in rate constant for distinct configurations in circularly excited FRET. Exact, full forms of these equations are provided in Supplementary Material. Notably, all results are expressible in terms of just two independent parameters, $\operatorname{Im} \gamma_{\mathrm{A}}$ and $\operatorname{Im} \gamma_{\mathrm{B}}$. 